\title{
Comparison of oral bioavailability of acetaminophen tablets, capsules and effervescent dosage forms in healthy volunteers
}

\author{
Mona Fathi ${ }^{1}$, Sohrab KaZemi ${ }^{1,2}$, Farbod Zahedi ${ }^{3}$, \\ Mohamad Reza Shiran ${ }^{4}$, Ali Akbar Moghadamnia ${ }^{1,2 *}$
}

${ }^{1}$ Neuroscience Research Center, Health Research Institute, Babol University of Medical Sciences, Babol, Iran

${ }^{2}$ Cellular and Molecular biology research centre, health research institute, Babol University of Medical Sciences, Babol, Iran

${ }^{3}$ Department of Pharmacology, Babol University of Medical Sciences, Babol, Iran

${ }^{4}$ Department of Pharmacology, Mazandaran University of Medical Science, Sari, Iran

\section{ARTICLE INFO \\ Received 17 November 2017 Accepted 20 December 2017}

\section{Keywords: \\ pharmacokinetics, \\ acetaminophen, \\ HPLC, \\ kinetic modeling, \\ effervescent.}

\begin{abstract}
A wide variety of acetaminophen dosage forms have been administered to relieve mild to moderate pain and fever, so far. The purpose of this study was to compare the oral bioavailability in healthy volunteers, of three of these dosage forms. We included healthy volunteers in our study and divided replace with placed them into three groups: tablet, capsule and effervescent. Each dosage form contained $500 \mathrm{mg}$ of acetaminophen as active material. Blood samples were taken at $0.5,1,2,4$ and 8 -hour intervals after receiving the dose. Acetaminophen blood levels were measured using HPLC method. Data were fit in a "one-compartment PK model", using P-Pharm 1.5 software and analyzed under statistical tests. The maximum concentrations of acetaminophen in blood samples were measured at $1 \mathrm{~h}$ after taking the drug $(6.61 \pm 3.19 \mu \mathrm{g} / \mathrm{ml}, 11.29 \pm 3.94 \mu \mathrm{g} / \mathrm{ml}$ and $15.25 \pm 2.54 \mu \mathrm{g} / \mathrm{ml}$ in groups receiving capsule, tablet and effervescent, respectively). Pharmacokinetic (PK) data analysis \& modeling from the three groups showed that the half-life of acetaminophen was $140.72 \mathrm{~min}$ in the tablet group, $140.29 \mathrm{~min}$ in capsule and $132.08 \mathrm{~min}$ in effervescent. The area under the blood levels curve were 47.04, 40.62 and $53.11 \mu \mathrm{gmin} / \mathrm{ml}$, in tablet, capsule, and effervescent groups, respectively. Statistically significant differences in PK parameters were recorded as the study replace with we compared effervescent with tablets and capsule dosage forms $(\mathrm{p}<0.05)$. According to the results, the effervescent form creates better PK parameters compared with tablet and capsule forms, therefore, it is suggested replace with we suggested that this form should be administer in cases of pain and fever to achieve quick drug efficacy.
\end{abstract}

\section{INTRODUCTION}

For years, methods to improve the efficacy of medications have been widely studied. It is known that the efficacy of a drug depends on its dosage form and route of administration [1]. Effervescent tablets are one of these forms that have drawn the attention of many for several reasons. Vitamin $\mathrm{C}$ tablets are probably the most well-known effervescents. However, several other medications are also made in this form nowadays [2]. These include Aspirin, antacids, iron, calcium and glucosamine supplements. These are all used in treating a wide range of conditions, from arthritis, pain and inflammation, to stomach and bowel problems, wounds, allergies and osteoporosis [3].

\footnotetext{
* Corresponding author

e-mail: aliamoghadamnia@gmail.com
}

Acetaminophen ( $\mathrm{N}$-acetyl para aminophenol, 4-hydroxyacetanilide) is absorbed quickly if administered orally [4]. However, the first pass effect reduces its access to proper circulation. It is an over-the-counter, non-narcotic drug which is widely used to relieve pain and reduce mild to moderate fevers. Although its mechanism of action has not been precisely worked out, studies have shown that the inhibition of central prostaglandin synthase plays an important role in the process. Acetaminophen does not inhibit the production of prostaglandins in the gastric mucosa [5], however, and, consequently, it can be helpful for patients with gastrointestinal disorders. It is also used as a part of combination therapy with other analgesics and anti-inflammatory agents. Several methods have been employed to make the absorption of acetaminophen tablets faster [6,7]. These include increasing 
the disintegration of the tablet, increasing the solubility of the drug using amino acid or alkali metal salts, and even adding sorbitol or antacids to acetaminophen tablets. The pharmacokinetics of various dosage forms of acetaminophen have been also broadly studied [8]; however, few studies have provided information on the amount of acetaminophen absorption in capsule formulation or the comparison of this amount between different formulations. Capsule and effervescent formulations are prepared of replace with can change acetaminophen to a more hydrophilic and more soluble powder [9]. The aim of this study was to assess the main pharmacokinetic properties of 3 formulations of acetaminophen (capsules, effervescents and tablets) based on the previously described acetaminophen powder and also to compare these features with all three accessible brand formulations of acetaminophen ( 2 conventional forms of solid tablets, effervescents and capsules).

\section{MATERIALS AND METHODS}

This study has been approved by the ethics committee of Babol University of Medical Sciences (Babol, Iran) and recorded in the data bank with a registration number. All subjects signed a written informed consent.

\section{Materials}

HPLC grade acetonitrile and water were purchased from Daejung (Daejung Chemicals Ltd., Korea). 4'-Methylacetophenone and Acetaminophen ( $>99.99 \%$ purity) were purchased from Merck Chemicals, (Merck KGaA, Darmstadt, Germany). Purified de-ionized water was prepared using the Milli Q50 water purification system (Millipore, USA).

\section{High-performance liquid chromatographic conditions}

The HPLC system was equipped with UV/VIS variable wavelength detector (KANUER, Germany), degasser article Nr. A5328 (KANUER Corporation, Germany). Acetophenone was used as internal standards (IS). Acetaminophen and (IS) were extracted using a mobile phase of acetonitrile and $0.1 \%$ acetic acid in water (20/80), via C18 column $(4.6 \mathrm{~mm} \times 25 \mathrm{~mm})$ and UV detector (wavelength $=240 \mathrm{~nm})$. Chromatographic resolution of Acetaminophen in serum was achieved on a $(4.6 \mathrm{~mm} \times 25 \mathrm{~mm}) \mathrm{C} 18$ column. Samples were then injected into HPLC with a syringe loading injector fitted with a $20 \mu$ loop.

\section{Preparation of Standard solutions}

An exact quantity of acetaminophen ( $5 \mathrm{mg}$ ) was transferred into a $5 \mathrm{ml}$ volumetric flask. Approximately $2.5 \mathrm{ml}$ of acetonitrile with $0.1 \%$ acetic acid (mobile phase) was then added and dissolved. To obtain a final concentration of 1.0 $\mathrm{mg} / \mathrm{ml}$, the solution was brought to volume by the mobile phase solvent and fully mixed. The prepared stock solution was kept at $4^{\circ} \mathrm{C}$ in a falcon vial. Standard solutions were freshly prepared prior to the analysis by stock solution.

\section{Linearity}

The calibration curves were produced with four concentrations ranging from 5 to $40 \mu \mathrm{g} / \mathrm{ml}$ of acetaminophen. Each concentration level was prepared and analyzed three times.
Thereafter, calibration curves were produced by plotting peak area response versus the concentration of compounds. The least square regression method was used for evaluation of linearity. The coefficient variation (CV) for this analysis was $3.8 \%$.

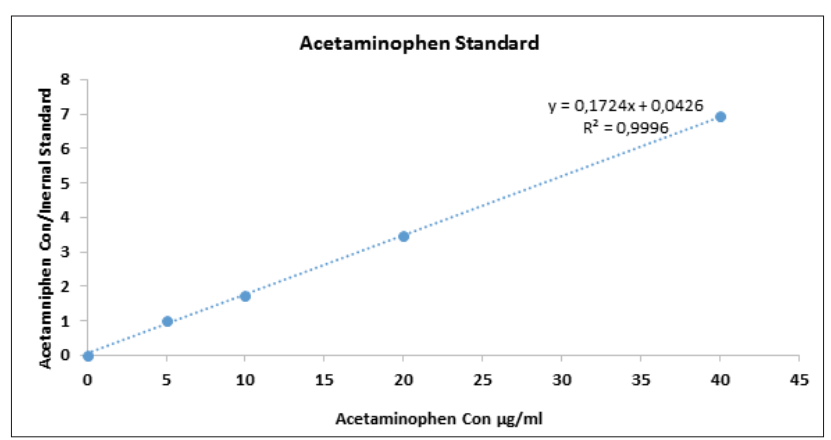

Figure 1. Least square regression method used for evaluating linearity

\section{The study procedure}

We enrolled 30 healthy volunteers in the study replace with into our study: 15 men and 15 women, with a mean $( \pm$ SD) age of $21( \pm 2)$. Participants were then placed within 3 groups, each containing 10 subjects, all of whom being prohibited to use acetaminophen or other analgesics one day before the experiment.

\section{Preparing samples}

For each participant, a sample of blood $(5 \mathrm{ml})$ was collected using an angiocatheter. The sample was obtained once before taking the drug by the subjects, and at $0.5,1$, $2,4,8 \mathrm{~h}$ after taking it, respectively. Blood samples were then collected in labeled, heparinized glass tubes and put into a $37^{\circ} \mathrm{C}$ water bath in order to separate serum contents. Thereafter, the samples were centrifuged in a refrigerated centrifuge $\left(4^{\circ} \mathrm{C}\right)$ for $10 \mathrm{~min}$ at $3000 \mathrm{rpm}$. They were then immediately transferred to a freezer and kept under $-20^{\circ} \mathrm{C}$ for later assays. To extract acetaminophen from the serum, $100 \mu \mathrm{l}$ of the serum sample was transferred to a $2 \mathrm{ml}$ vial containing $20 \mu \mathrm{l}$ 4-methylacetophenone as internal standard. A volume of $500 \mu \mathrm{l}$ of methanol was added to the sample afterwards. The vial was then vortexed for $1 \mathrm{~min}$ and the solution that remained on top was separated using a sampler. Subsequently, $100 \mu \mathrm{l}$ of solvent was stored with an open cap in a $4^{\circ} \mathrm{C}$ fridge. A volume of $20 \mu \mathrm{l}$ of final extracted solution was directly injected to HPLC (Figure 2).

The pharmacokinetic (PK) parameters such as absorption rate constant $(\mathrm{Ka})$, clearance, half-life and volume of distribution of acetaminophen in three groups were calculated based on one-compartment kinetics using P-Pharm software $(10,11)$. According to data distribution (Table 1), the results were analyzed using analysis of variance. The difference of the PK parameters between three groups was considered statistically significant at $\mathrm{p}<0.05$.

\section{Statistical analysis}

According to the results, On-way ANOVA followed by tuky post-hoc test was used. The differences of the PK parameters of three groups were analyzed and considered statistically significant at $\mathrm{P}<0.05$. 
Table 1. Comparison of the PK parameters of three groups receiving Acetaminophen

\begin{tabular}{|c|c|c|c|c|c|}
\hline $\begin{array}{c}\text { Fold } \\
\text { changes }\end{array}$ & Min & Max & Mean & Formulation & \begin{tabular}{|c|}
$\mathrm{Pk}$ \\
parameters \\
\end{tabular} \\
\hline 1.38 & 0.11 & 0.16 & 0.14 & Eff & \multirow{3}{*}{$\mathrm{Cl}(\mathrm{L} / \mathrm{min})$} \\
\hline 2.29 & 0.11 & 0.25 & 0.17 & Cap & \\
\hline 1.20 & 0.13 & 0.16 & 0.14 & Tab & \\
\hline 1.11 & 24.64 & 27.47 & 25.88 & Eff & \multirow{3}{*}{$V / F(L)$} \\
\hline 1.46 & 24.65 & 36.05 & 32.87 & Cap & \\
\hline 1.30 & 24.49 & 31.81 & 28.46 & Tab & \\
\hline 2.89 & 0.03 & 0.09 & 0.06 & Eff & \multirow{3}{*}{$\mathrm{Ka}(1 / \mathrm{min})$} \\
\hline 9.15 & 0.01 & 0.07 & 0.05 & Cap & \\
\hline 2.26 & 0.01 & 0.02 & 0.01 & Tab & \\
\hline 1.29 & 120.28 & 154.94 & 132.08 & Eff & \multirow{3}{*}{$\mathrm{t} 1 / 2(\mathrm{~min})$} \\
\hline 2.46 & 91.70 & 225.98 & 140.29 & Cap & \\
\hline 1.27 & 119.75 & 152.40 & 140.72 & Tab & \\
\hline
\end{tabular}

Tmax: maximum time to maximum concentration, Cmax: maximum concentration, MIN: minimum concentration, MAX: maximum concentration, F: folding of maximum to minimum concentration or MAX divided by MIN, Eff: effervescent, Cap: capsule, Tab: tablet

\section{RESULTS}

\section{Acetaminophen concentrations}

Comparison of acetaminophen concentrations shows a statistical difference between the three groups $(\mathrm{p}<0.05)$; Table 2 and Figure 3 show serum concentrations of effervescents in comparison with the other two groups. In the effervescent group, the highest concentration was observed at 60 min after dosing. The concentration then increased rapidly to the maximum level after $60 \mathrm{~min}$, decreased gradually and reached its lowest point at $480 \mathrm{~min}$. The maximum and minimum concentrations of acetaminophen were 15.24 and $1.49 \mu \mathrm{g} / \mathrm{ml}$ for the effervescent, 11.29 and $2.08 \mu \mathrm{g} / \mathrm{ml}$ for the capsule and 8.73 and $2.64 \mu \mathrm{g} / \mathrm{ml}$ for tablet forms, respectively (Table 2).

Table 2. Comparison of the Area under the curve of three groups receiving Acetaminophen

\begin{tabular}{|c|c|c|c|}
\hline \multicolumn{4}{|c|}{ Observed plasma concentration $(\mu \mathrm{g} / \mathrm{ml})$} \\
\hline Tablet & Capsule & Effervescent & Time (min) \\
\hline 0 & 0 & 0 & 0 \\
\hline $6.61 \pm 2.41$ & $11.29 \pm 3.94$ & $15.25 \pm 2.54$ & 60 \\
\hline $8.74 \pm 2.49$ & $7.43 \pm 1.37$ & $9.94 \pm 1.63$ & 120 \\
\hline $7.35 \pm 2.80$ & $4.67 \pm 1.49$ & $6.67 \pm 1.81$ & 240 \\
\hline $2.64 \pm 0.85$ & $2.08 \pm 1.70$ & $1.49 \pm 0.64$ & 480 \\
\hline 47.04 & 40.62 & 53.11 & $\Sigma$ AUC \\
\hline
\end{tabular}

\section{Standard curve}

To determine the concentration of acetaminophen in serum, a standard curve was plotted with standard solution of the drug containing $100 \mu \mathrm{g} / \mathrm{ml}$ of acetaminophen prepared in methanol. Of note, 4'-methylacetophenone was added as internal standard for all concentrations (Figure 1). Standard peaks of acetaminophen of 5, 10 and $20 \mu \mathrm{g} / \mathrm{ml}$ and internal standard $(10 \mu \mathrm{g} / \mathrm{ml})$ were prepared. A standard curve was obtained using the peak area under the curve (Figure 2). Moreover, R2 index (linearity) was depicted in the chart.
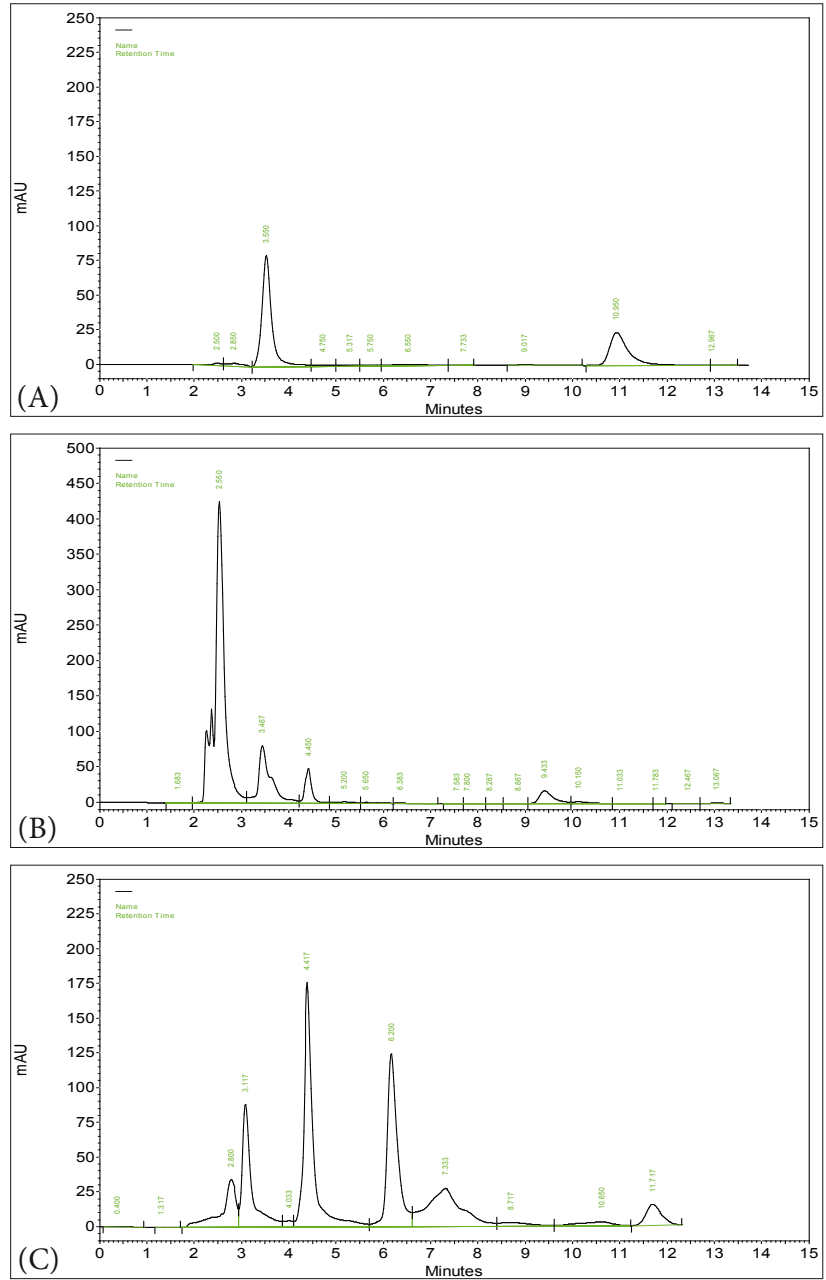

Figure 2. The Sample peaks of acetaminophen, $(3.4 \mathrm{~min})$ (A) standard acetaminophen $(10 \mu \mathrm{g} / \mathrm{ml})$; (B) serum sample after $2 \mathrm{~h}$ acetaminophen administration; (C) serum Sample after $1 \mathrm{~h}$ acetaminophen administration

\section{Data analysis with P-Pharm}

We used P-Pharm software to model and analyze the obtained data on serum concentrations of the drug. According to the modeling of serum concentrations of acetaminophen versus time, the results of oral administration of threecompartment kinetics on the PK modeling are shown below:

A) Concentration versus time curve in tablet group: Figure $3 \mathrm{~A}$ shows the correlation between concentrations versus time for any subject in the tablet group. This figure shows only two scattered levels at $120 \mathrm{~min}$.

B) Concentration versus time curve in capsule group: Figure 3B shows a correlation replace with regression between concentrations versus time of the individuals in the capsule group. This figure demonstrates scattered concentrations at 60 and 240 min after drug administration. The maximum concentration of acetaminophen in serum samples was recorded $60 \mathrm{~min}$ after oral administration (Figure 3B).

C) Concentration versus time curve in effervescent group: Figure $3 \mathrm{C}$ indicates a correlation replace with regression between concentrations versus time of the subjects in the effervescent group. In this figure, scattered concentrations are visible at $60 \mathrm{~min}$ after drug consumption. 

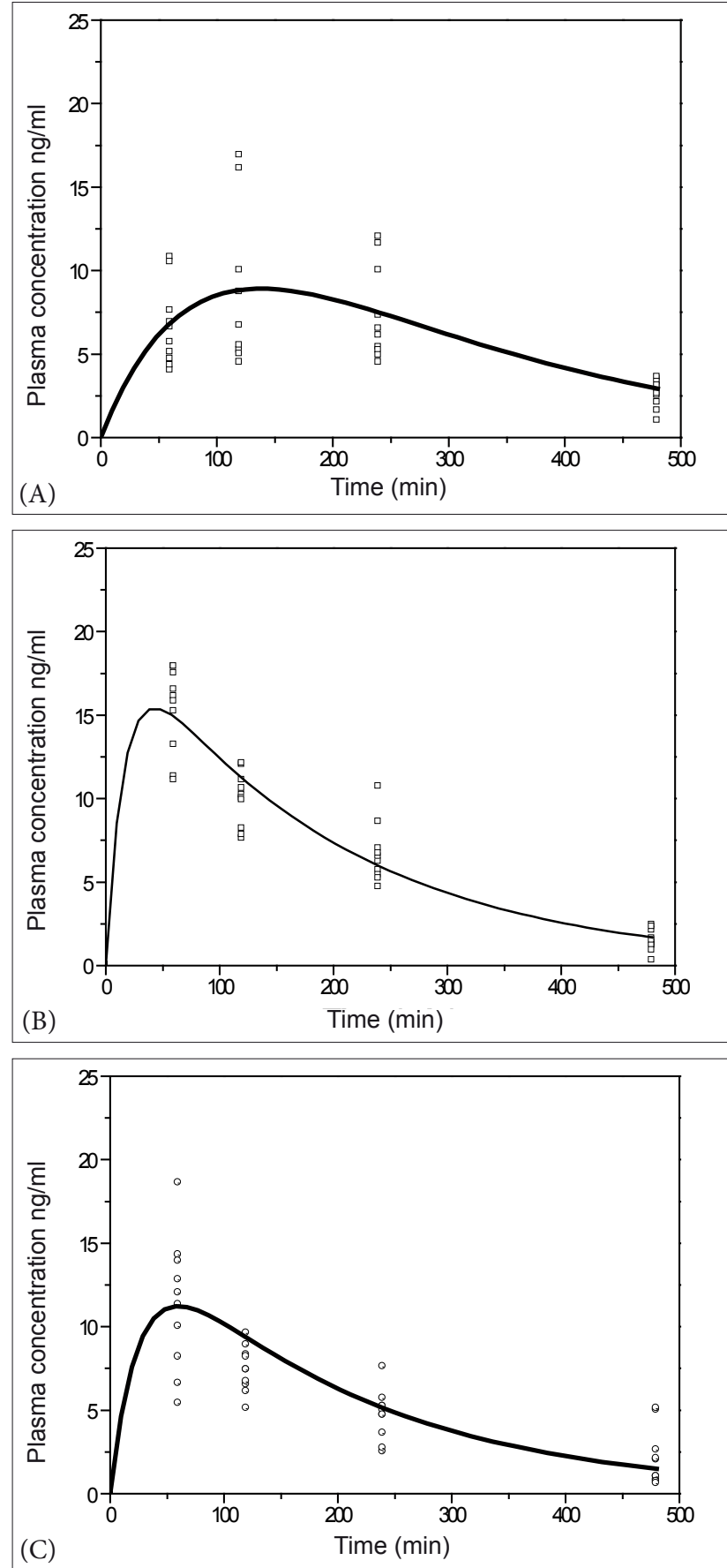

Figure 3. The relationship between acetaminophen concentrations vs. time in each subject (individual fitting) in A Tablet, B Capsule and C Effervescent groups after PK modeling based on Three compartment Oral administration kinetics. A Krukenberg tumor is a metastatic adenocarcinoma of for proper treatment

Figure 4 shows that in the effervescent and capsule groups, maximum concentration of acetaminophen is achieved at $60 \mathrm{~min}$, while this amount of time will rise to $120 \mathrm{~min}$ for the tablet group. There were no significant changes in participants' vital signs or biochemical findings. What is more, there were no cases of mild dyspepsia following the administration of each formulation. In general, treatment with acetaminophen $(500 \mathrm{mg})$ has shown itself to be safe and well-tolerated in healthy volunteers.

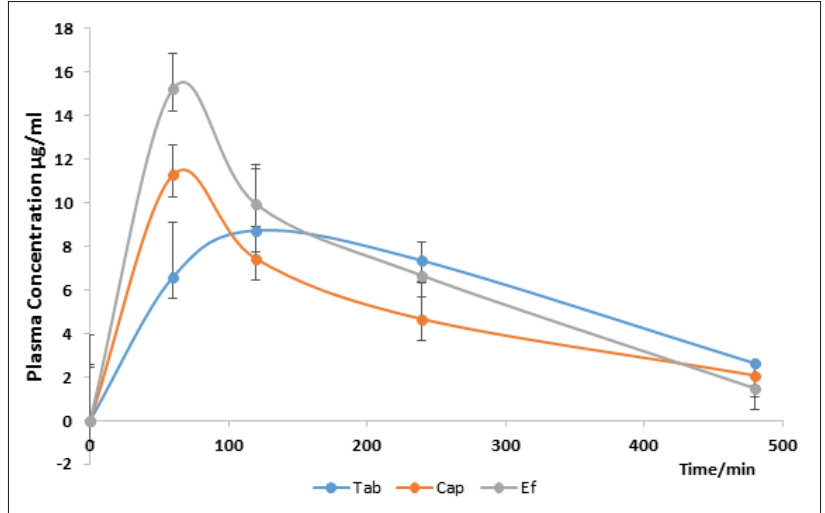

Figure 4. Mean of plasma concentration - time profiles of acetaminophen in the three groups of the study after a single 500 $\mathrm{mg}$ oral dose of acetaminophen from different dosage forms

\section{DISCUSSION}

Salt formation has a key role to increase solubility and absorption of acetaminophen [10] without any changes in chemical structure and pharmacological properties. Among different formulations of acetaminophen, the effervescent dosage form has a quicker absorption (Greater $\mathrm{C}_{\max }$ and lower $\mathrm{T}_{\max }$ ) (Table 2). The area under the plasma concentration curve, however, shows that tablet and capsule forms were absorbed much more than the effervescent during the first hour of administration and the AUC of effervescent group is significantly higher than the two other formulations.

When the goal is to relieve pain in a faster and more efficient way, it is better to use soluble or quickly-absorbed formulations because delayed absorption or low concentrations of the drug may result in treatment failure. Another issue that has always been of great importance for pharmacists is to prevent drug alterations that occur in the GI system [11]. Such changes may occur due to the low $\mathrm{pH}$ of the stomach and food/drug interactions, causing the drug to be inactivated. With their buffering properties, effervescents increase gastric $\mathrm{pH}$ and therefore prevent active drug forms from dissociation and inactivation [12]. This buffering feature (carbonation) of effervescents helps the stomach to empty itself quickly. It usually takes $20 \mathrm{~min}$ for gastric components to reach the small intestine, therefore, the same amount of time is required for activated drugs to reach maximum absorption [13].

In all the 3 groups, we observed that the serum concentrations of the drug reached their maximum at the first hour after administration. Our study also found that capsules and effervescents equally reached the maximum concentration for acetaminophen, while it took $120 \mathrm{~min}$ for tablets to reach this concentration after a single dose of the drug. In addition, effervescents had the highest serum concentrations during the first hours of administration - more than 2.5 times greater than tablets.

Studies have shown that compared with tablets, effervescents better improve the absorption rate of several activated drugs (e.g caffeine and disulfiram), simply because the $\mathrm{CO}_{2}$ resulting from acid-base reactions can increase their permeability [14]. Given that the drug component of effervescents enters the GI system as a recently produced solution [15], 
they are absorbed at a higher rate than other formulations. In addition, when effervescents dissolve in water, the $\mathrm{pH}$ of the final solution causes the drug to transit faster from stomach to small intestine. Effervescents also last longer than liquid drug forms such as syrups or suspensions [16].

\section{CONCLUSIONS}

The three reasons mentioned above indicate that effervescents have a quicker effect compared with tablets. We can claim that the absorption of the drug component of effervescents is faster than tablets and a faster absorption may suggest a faster effect.

\section{ACKNOWLEDGMENTS}

The authors thank Dr. Reza Ghadimi for his cooperation. This investigation was supported financially by the Research Affairs Division of Babol University of Medical Sciences.

\section{CONFLICT OF INTERESTS}

The authors declare that they have no competing interests.

\section{REFERENCES}

1. Santovena A, et al.: Formulation design of oral pediatric Acetazolamide suspension: dose uniformity and physico-chemical stability study. Pharm. Dev. Techn. 2017;22:191.

2. Ubeda A, et al.: Blood pressure reduction in hypertensive patients after withdrawal of effervescent medication. Pharmacoepidemiol Drug Saf.2009;18:417.
3. Ferreira NN, et al.: A novel pH-responsive hydrogel-based on calcium alginate engineered by the previous formation of polyelectrolyte complexes (PECs) intended to vaginal administration. Drug Dev Ind Pharm. 2017;1.

4. Koen YM, et al.: Comparative Toxicity and Metabolism of N-Acyl Homologues of Acetaminophen and Its Isomer 3-Hydroxyacetanilide. Chem Res Toxicol 2016;29:1857.

5. Anderson BJ: Paracetamol (Acetaminophen): mechanisms of action. Paediatr Anaesth. 2008;18:915.

6. Preis M: Orally disintegrating films and mini-tablets-innovative dosage forms of choice for pediatric use. AAPS PharmSciTech. 2015; $16: 234$

7. Alam MT, et al.: FDA-Approved Natural Polymers for Fast Dissolving Tablets. J Pharm (Cairo). 2014;952970.

8. Rawlins M, et al.: Pharmacokinetics of paracetamol (acetaminophen) after intravenous and oral administration. Eur J Clin Pharm. 1977;11:283.

9. Srinath K, et al.: Formulation and evaluation of effervescent tablets of paracetamol. INT J Pharm Res Dev. 2011;3:76.

10. Blagden $\mathrm{N}$, et al.: Crystal engineering of active pharmaceutical ingredients to improve solubility and dissolution rates. Adv Drug Deliv Rev 2007 Jul 30;59(7):617-30 Epub 2007 May 29.

11. De Silva L, et al.: Usability of mobile technology to screen for drugdrug interactions in kidney transplant patients. Am J Nephrol. 2014;40(2):97-104. doi: 101159/000364910 Epub 2014 Jul 19.

12. Moes AJ: Gastroretentive dosage forms. Crit Rev Ther Drug Carrier Syst. 1993;10(2):143-95.

13. Wilding II, et al.: Development of a new engineering-based capsule for human drug absorption studies. Pharm Sci Technol Today. 2000;3:385.

14. Lopes CM, et al.: Overview on gastroretentive drug delivery systems for improving drug bioavailability. Int J Pharm. 2016;510:144.

15. Altman R, et al.: Advances in NSAID development: evolution of diclofenac products using pharmaceutical technology. Drugs. 2015;75:859.

16. Sohi $\mathrm{H}$, et al.: Taste masking technologies in oral pharmaceuticals: recent developments and approaches. Drug Dev Ind Pharm. 2004;30:429. 